\title{
Comparative Study of Degree of Low Grade Chronic Inflammatory Status in Freshly Diagnosed Type 1 with Type 2 Diabetic Patients
}

\author{
Shamim Shaikh Mohiuddin ${ }^{1 *}$ and Poornima Manjrekar ${ }^{2}$ \\ ${ }^{1}$ Department of Biochemistry, College of Medicine, Imam Abdulrahman Bin Faisal University, Kingdom of Saudi Arabia
}

${ }^{2}$ Department of Biochemistry, kasturba Medical College, Manipal University, Mangalore, India

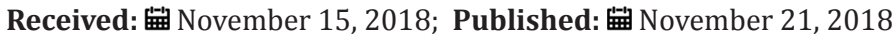

*Corresponding author: Shamim Shaikh Mohiuddin, Department of Biochemistry, College of Medicine, Imam Abdulrahman Bin Faisal University, Kingdom of Saudi Arabia

\begin{abstract}
Low grade inflammatory condition which is predominately cytokine-mediated is observed to be closely involved in the pathogenesis of type 2 diabetes as well as role this inflammatory condition cannot be ruled out in involvement in pathogenesis of type 1 diabetes. Since maximum world populations are at high risk of developing diabetes, we tested this hypothesis by estimating circulating acute phase proteins in freshly diagnosed type 2 (T-2) as well as type1(T-1) diabetic patients.

The acute phase proteins, $\alpha 1$ - antitrypsin, $\alpha 1$ - acid glycoprotein, ceruloplasmin and fibrinogen were estimated in the plasma in freshly diagnosed twenty-five T-2 cases and twelve T-1cases. Thirty normal controls to match the age and sex of the test groups were also studied. The levels of these proteins were correlated with their BMI and random plasma glucose values.

In comparison with the controls, the values of all the four proteins studied were significantly elevated in the T- 2 patients $(p<00001)$. In case of T-1 patient level of ceruloplasmin and fibrinogen are significantly elevated. Interestingly, no correlation was found with BMI or the degree of hyperglycemia in either of the types. A low-grade inflammatory process is definitely implicated in the pathogenesis of type 2 diabetes. Same can be ruled out in type-1 diabetic patients also. The comparative study of the states of all these inflammatory markers in case of T- 2 and T-1 patients is of interest in this present study.
\end{abstract}

Keywords: Acute Phase Proteins; Low Grade Chronic Inflammation; Type 1 Diabetes Mellitus; Type 2 Diabetes Mellitus

Abbreviations: CRP: C-reactive Proteins; IL-1: Interleukin-1; IL-6: Interleukin-6; TNF: Tumor Necrosis Factor; RBS: Random Blood Sugar; LDL: Low Density Lipoprotein; KD: Kilo Dalton

\section{Introduction}

Diabetes Mellitus is one of the most common major public health problems having worldwide distribution. It has now adopted epidemic proportions. It is estimated that by 2025, an estimated 300 million people will have diabetes, most of whom will inhabit of China, India and United States [1] Although hyperglycemia is the main characteristic of all form of diabetes mellitus, the pathogenic mechanism by which hyperglycemia arises differs widely. Some forms of Diabetes mellitus are characterized by an absolute insulin deficiency or a genetic defect leading to defective insulin secretion; where as other forms share insulin resistance as their underlying etiology. The two broad categories of Diabetes mellitus are designated as type 1 and type 2 . The type 1 Diabetes mellitus results from autoimmune B cell destruction which usually leads to insulin deficiency. Type 2 Diabetes mellitus is a heterogeneous

group of disorders usually characterized by variable degree of insulin resistance, impaired insulin secretion and increased glucose production.

Recently, there in increasing evidence that an ongoing cytokine induced acute phase response which is sometimes called low grade inflammation, but part of a widespread activation of the innate immune system, is closely involved in the pathogenesis of type 2 diabetes mellitus and associated complications such as dyslipidemia and atherosclerosis. Elevated circulatory inflammatory markers such as C-reactive protein and interleukin-6 predict the development of type 2 Diabetes mellitus. Age, inactivity, certain dietary components, smoking, psychological stress and low birth weight are among the risk factors for type 2 diabetes mellitus, which are also known to be associated with activated innate 
immunity. Activated immunity may be the common antecedent of developing type 2 diabetes mellitus. Other features of type 2 diabetes mellitus such as fatigue, sleep disturbance and depression are likely to be at least partly due to hypercytokinemia and activated innate immunity [2]. $\alpha 1$ - acid glycoprotein, $\alpha-1$ antitrypsin, fibrinogen and ceruloplasmin are few of the acute phase reactants. The level of these inflammatory markers in the pathogenesis of type 1 and type 2 Diabetes mellitus was of interest.

\section{Aims and Objectives}

The aims of the present study are

a. To detect the elevation of plasma level of $\alpha 1$ - antitrypsin, $\alpha 1$ acid glycoprotein, ceruloplasmin and fibrinogen as an inflammatory marker (acute phase reactant), if any, in newly diagnosed untreated type 1 diabetes mellitus patients as well as in newly diagnosed untreated type 2 diabetes mellitus patients.

b. Compare the level of $\alpha 1$ - antitrypsin, $\alpha 1$ - acid glycoprotein, ceruloplasmin and fibrinogen in newly diagnosed untreated type 1 diabetes mellitus with newly diagnosed untreated type 2 diabetes mellitus patients.

\section{Material and Methods}

\section{Study Design}

Following groups of subjects were selected for present study:

a. Twelve newly diagnosed untreated type 1 diabetes mellitus patient

b. Twenty-five newly diagnosed untreated type 2 diabetes mellitus patients within the age limit of 30-60 years

c. Thirty nondiabetic healthy controls.

Height and weight of all subjects were recorded, and body mass index was calculated. Patients with features of previously undiagnosed diabetes were examined by the physicians. Of the confirmed cases, patients with a history of chronic inflammatory diseases, episodes of recent acute inflammation, smokers, alcoholics, women on oral contraceptive pills or any other hormones, pregnant women and patients with clinical evidence of neuropathy, nephropathy, and retinopathy were not enrolled in the study. Twenty-five (25) type 2 patients and twelve (12) type 1 patients of either sex gave their consent to participate in the study. Type 2 was decided based only on their age and their subsequent response to insulin and oral hypoglycemic, respectively. Thirty (30) individuals were chosen from attendants of the patients to serve as controls. All exclusion criteria of the test groups were applied to the control group also. The study was undertaken in Kasturba Medical College, Mangalore, India and was approved by the Institutional Ethics Committee. Age, weight and height were recorded and body mass index (BMI) was calculated. Blood was collected as a random sample before the initiation of therapy in the diabetic patients and the following estimations were carried out: a. Random plasma glucose (RBS): By the glucose oxidase method on Hitachi 917 autoanalyser using Roche Kits.

b. Fibrinogen assay [3]: Fibrinogen in plasma was converted to fibrin in the presence of calcium chloride. The fibrin clot was collected and digested with sodium hydroxide. Protein content of the clot was determined by the biuret method.

c. Ceruloplasmin assay [4]: At pH 5.4, ceruloplasmin catalysis the oxidation of paraphenylenediamine (PPD) to yield a coloured product which is believed to correspond either to Bandrowski's base or to Weuster's red. The rate of formation of the coloured oxidized product is proportional to the concentration of ceruloplasmin, if a correction is made for the nonenzymatic oxidation of PPD. Simultaneous estimations were carried out with and without sodium azide, which inhibits the nonenzymatic oxidation of PPD. The difference between the results of the two assays was proportional to the ceruloplasmin concentration.

d. $\boldsymbol{\alpha}-\mathbf{1}$ antitrypsin assay [5]: The proteolytic enzyme trypsin hydrolyses casein, with the formation of smaller peptides. The enzyme reaction after suitable interval of time is arrested by the addition of tri-chloroacetic acid (TCA) which precipitates the proteins, but the peptides are soluble in the acid. The TCA soluble fragments are a measure of proteolytic activity of this enzyme. When the inhibitor is added to the preincubated mixture, it prevents the release of peptides by the proteolytic enzymes. Thus, the estimation of TCA soluble components in the presence and absence of inhibitor is a measure of inhibitory activity against proteolytic enzymes. The TCA soluble fragments were analyzed by the method of Lowry et al [6]. The final color formed is a result of the reaction of the peptides with copper ions in alkali and reduction of the phosphomolybdic reagent by the presence of tyrosine and tryptophan present in the treated peptides.

e. $\boldsymbol{\alpha}-\mathbf{1}$ acid glycoprotein [7]: After removing heat coaguable proteins with perchloric acid, the orosomucoid which remains in the solution was precipitated by phosphotungstic acid and estimated by determining its carbohydrate content by reaction with its tyrosine residues with folin ciocalteau reagent. Statistics The data was analyzed by the students t test and the ANOVA test. Pearson's coefficient was applied for correlational analysis.

\section{Statistics}

The data was analyzed by the students' t test and the ANOVA test. Pearson's coefficient was applied for correlational analysis.

\section{Results}

By the entire study our aim was to determine whether low grade chronic inflammation with associated activated innate immune system is a pathogenic cause in freshly diagnosed type 2 diabetes mellitus cases or not. Even we studied the association of this chronic inflammatory response as well as activated innate immune system in freshly diagnosed type 1 diabetic patients (Table 1). 
Table 1: Characteristics of subjects.

\begin{tabular}{|c|c|c|c|}
\hline & Controls (n= 30) & Type 2 (n= 25) & Type 1(n=12) \\
\hline Age & $43.97 \pm 14.06$ & $47.27 \pm 7.11$ & $18.33 \pm 7.64$ \\
\hline BMI & $20.75 \pm 2.27$ & $23.03 \pm 1.46$ & $19.50 \pm 1.23$ \\
\hline
\end{tabular}

$\mathrm{n}=$ number of subjects

\section{Discussion}

This study was setup for detection whether pathogenicity of type 2 as well as type 1 diabetes mellitus is related with low grade chronic inflammatory process with activated innate immunity or not and if the answer is yes then what are the comparative values of inflammatory markers between these two types of diabetes. In the twelve newly diagnosed type 1 patients, the level of $\alpha 1$-antitrypsin, ceruloplasmin and fibrinogen were found to be significantly increased as compared to control (Table 2). Previous reports on the acute phase reactant levels in Type 1 diabetes are contradictory. Crooke MA et al. [8] has shown that serum sialic acid and acute phase proteins are not elevated in type 1 diabetes. Gomes et al. [9] reported increased level of CRP, $\alpha 1$-acid glycoprotein and fibrinogen in Type 1 patients. Increased fibrinogen levels, factor VII and whole blood viscosity was also found by John AD Elia et al. [10]. Similar results are reported by Defeo et al. [11]. In our study, an increase in $\alpha 1$-antitrypsin, ceruloplasmin and fibrinogen levels were found in type 1 patients.

Table 2: Values of the acute phase proteins as Mean \pm SD.

\begin{tabular}{|c|c|c|c|}
\hline Parameters & Controls Mean \pm SD & Type 2 Mean \pm SD & Type 1 Mean \pm SD \\
\hline Random blood Sugar (mg/dL) & $93.20 \pm 7.00$ & $192.26 \pm 35.20$ & $338.25 \pm 50.97$ \\
\hline$\alpha 1$ antitrypsin $(\mathrm{mg} / \mathrm{dL})$ & $349.48 \pm 114.07$ & $561.16 \pm 63.00$ & $495.70 \pm 32.77$ \\
\hline$\alpha 1$ acid glycoprotein(mg/dL) & $102.41 \pm 22.13$ & $180.93 \pm 31.94$ & $94.87 \pm 23.31$ \\
\hline Ceruloplasmin (mg/dL) & $25.95 \pm 4.10$ & $44.05 \pm 9.03$ & $40.69 \pm 9.85$ \\
\hline Fibrinogen $(\mathrm{mg} / \mathrm{dL})$ & $334.34 \pm 42.19$ & $571.25 \pm 82.26$ & $434.65 \pm 46.36$ \\
\hline
\end{tabular}

Twenty-five type 2 newly diagnosed patients showed increased levels of $\alpha 1$-antitrypsin, $\alpha 1$-acid glycoprotein, ceruloplasmin and fibrinogen (Table 2). The findings were in agreement with most of the authors who worked with acute phase proteins in type 2 diabetes [12,13]. The role of chronic low-grade inflammation in the pathogenesis of type 2 diabetes seems possible beyond doubt. At the same time its role in type 1 diabetes cannot be completely ruled out. The course of the disease and resulting complications are similar in both type 1 and type 2 diabetes. The most dreaded complication being that of development of atherosclerosis resulting in cardiovascular diseases. Fibrinogen is identified as an independent risk factor in the development of ischemic heart diseases [14]. Irrespective of the patients being type 1 or type 2, the risk of developing atherosclerosis remains the same. Hence there must be some mechanism which links the pathogenicity of type 1 and type 2 diabetes. Barrazzani R et al. [15] infused insulin to non-diabetics, type 1 and type 2 diabetics and studied its role in fibrinogen production. Insulin replacement activity suppressed fibrinogen production in non-diabetics and type 1 diabetic individuals.

Fibrinogen production and its plasma concentration increased in insulin resistant type 2 diabetics when euglycemia and euaminoaciduria were maintained. They postulated that an altered response to insulin causes hyperfibrinogenemia in type 2 diabetic patients. If this hypothesis holds well, it doesn't explain hyperfibrinogenemia in type 1 diabetics where the basic pathology is insulin deficiency. Hence there must be some other factors which stimulate increased fibrinogen synthesis in type
1 patients contributing to cardiovascular disease risk. An insulin resistance syndrome score [16] was developed based on clinical risk factor in patient with type 1 diabetes and validated using euglycemic-hyperinsulinemic clamp studies. Fibrinogen levels were significantly associated with this insulin resistance syndrome score. This may explain high fibrinogen level in type 1 diabetes. But it still does not answer the above findings since the type subjects in this study were newly diagnosed. Hence the mechanism of increased fibrinogen synthesis needs to be proved further.

Ceruloplasmin is known to have antioxidant action. It is also an acute phase protein with a response of intermediate magnitude. Ceruloplasmin is known to stimulate cell proliferation and angiogenesis. The higher levels of ceruloplasmin in both in type 1 and type 2 as compared to controls may be due to an oxidative stress that is prevalent in both types of diabetes. Eduardo Ehrenwald showed a very interesting feature of ceruloplasmin. The intact human ceruloplasmin which is $132 \mathrm{kDa}$ molecules caused increased oxidation of LDL in vitro [17]. Starkebaum G and Harlan JM et al also showed that increased serum ceruloplasmin could generate excess oxidized LDL and cause vascular injury by generating free radicals such as hydrogen peroxide [18]. These findings defined the earlier notions of the antioxidant activity of ceruloplasmin. By further investigations Eduardo Ehrenwald et al found that the holoceruloplasmin, which is seen in serum as a 132 $\mathrm{kDa}$ molecule, has a prooxidant effect and the action was attributed to the copper ions present in ceruloplasmin.

The commercially available ceruloplasmin is a degraded product containing either $115 \mathrm{kDa}$ fragment or [19] kDa fragment 
or both. These had an antioxidant effect. The works done to show that ceruloplasmin as an antioxidant used these degraded products. The antioxidant action of a commercial ceruloplasmin was observed even in the system where holoceruloplasmin oxidized LDL. Hence considering ceruloplasmin as an antioxidant in vivo is debatable. The LDL oxidizing action of ceruloplasmin could probably explain at least in part of the increased risk of IHD in both type 1 and type 2 diabetes. Also, it could not be wrong to count ceruloplasmin as an acute phase reactant whose levels are increased in both the types of diabetes. The values of various parameters when compared between the untreated type 1 patients and type 2 patients reveals a significant increase in type 2 patients (Table 2 ).

Even the ceruloplasmin values, although not statistically significant, were slightly higher in the type 2 patients. The mean random blood sugar (RBS) values in group 1(Type 1 diabetes) patients was $338.25 \pm 0.97 \mathrm{mg} / \mathrm{dl}$ and that of group II (Type 2 newly diagnosed diabetics) was $193.26 \pm 35.30 \mathrm{mg} / \mathrm{dl}$. Despite this huge difference, the inflammatory markers levels were higher in the type 2 patients which go to prove that the glycemic status doesn't influence the inflammatory markers. This is in accordance with previous findings [19]. Evidence is available to say that inflammatory markers are elevated well before the clinical manifestation of hyperglycemia [20-23]. This also gives credence to the thought that activation of innate immunity is not related to hyperglycemia. But research has shown that decreasing plasma glucose levels decrease the concentration of acute phase reactants. Also $2 \mathrm{hrs}$ post load glucose values showed positive correlation with the inflammatory markers in few studies [24]. $\alpha 1$-acid glycoprotein levels remained within normal limits in the type 1 patients whereas significant high levels were seen in the type 2 patients $(\mathrm{p}<0.0001)$.

Table 3: Comparison of $\mathrm{p}$ values between groups.

\begin{tabular}{|c|c|c|c|}
\hline Parameters & T-2 v/s Controls & T-1 v/s Controls & T-2 v/s T-1 \\
\hline Random blood Sugar (mg/dL) & $<0.0001^{*}$ & $<0.0001^{*}$ & $<0.0001^{*}$ \\
\hline$\alpha 1$ antitrypsin(mg/dL) & $<0.0001^{*}$ & $0.0002^{*}$ & 0.003 \\
\hline$\alpha 1$ acid glycoprotein (mg/dL) & $<0.0001^{*}$ & 0.275 & $<0.0001^{*}$ \\
\hline Ceruloplasmin (mg/dL) & $<0.0001^{*}$ & $<0.0001^{*}$ & 0.19 \\
\hline Fibrinogen $(\mathrm{mg} / \mathrm{dL})$ & $<0.0001^{*}$ & $<0.0001^{*}$ & $<0.0001^{*}$ \\
\hline
\end{tabular}

T-2= Type 2 newly diagnosed patient

$\mathrm{T}-1=$ Type 1 newly diagnosed patient

$\mathrm{p} \leq 0.05$ was considered as significant

*= statistically significant

Although both type 1 and type 2 patients showed significant high $\alpha 1$-antitrypsin levels (Table $2 \& 3$ ), the comparison between two groups also reached statistical significance $(p=0.003)$ with higher values in type 2 patients. The above findings can be best explained as insulin mediated increased synthesis of the hepatic proteins. The underlying mechanism for the augmented acute phase response is not well understood and the stimulus for the response is unknown. A number of hypotheses have been put forward and these include insulin resistance, obesity, atherosclerosis, other diabetic complications and maladaptation of the normal innate immune response to environmental threats [25-27].

The most widely studied is the association of obesity, insulin resistance, type 2 diabetes and acute phase reactants. It has been shown that adipocytes secret a number of proinflammatory cytokines in the postprandial state [28-30]. The term 'diabecity' has received attention [31] of late for obese diabetics. The 'common soil' theory proposed, evaluates the involvement of inflammation in the pathogenesis of diabetes and atherosclerosis. Hyperglycemia and insulin resistance could promote inflammation and inflammation may be a factor linking diabetes mellitus to the development of atherosclerosis. Elevated glucose levels promote inflammation by increasing oxidative stress [32], by the formation of AGEs and

increased TNF (kappa B) [33]. In this study, the mean BMI was found to be $19.5 \pm 1.23$ in type 1 patient and $24.03 \pm 1.46$ in type 2 patients. No correlation was found between BMI and acute phase reactants. Hence it can be summarized that there could be multiple pathways involved in the activation of the innate immunity system and much work needed to be done to establish either a casual role in the development of mainly type 2 diabetes and could be type 1 diabetes also.

\section{References}

1. Abate N, Chandalia M (2001) Ethnicity and type 2 diabetes: focus on Asian Indian. J Diabetes Complications 15(6): 320-327.

2. Pickup JC (2004) Inflammation and activated innate immunity in the pathogenesis of type 2 diabetes. Diabetes care 27(3): 813-823.

3. Varley H, Gowenlock AH, Bell M (1991) Determination of plasma fibrinogen. In: Practical Clincial Biochemistry. CBS publishers and distributors ( $5^{\text {th }}$ edn.). pp. 557-1559.

4. Sunderman Jr FW, Nomoto S (1970) Measurement of human serum ceruloplasmin by its p-phenylenediamine oxidase activity. Clinical Chemistry 16(11): 903-910.

5. Sundaresh CS, Aroor AR, Pattabiraman TN (1978) Comparative study of amidolytic and caseinolytic methods for the determination of urinary trypsin inhibitor. Indian J Med Res 68: 341-334.

6. Loway OH, Rosebaugh NJ, Farr AL, Randall RJ (1951) Protein measurement with folin phenol reagent. J Biol Chem 193: 265-275. 
7. Winzler RJ, et al. (1955) Determination of serum $\alpha-1$ acid glycoprotein. In: Methods in Biochemical Analysis. Inter-science Pub, New York, USA 2: 270 .

8. Crook MA, Tutt P, Simpson H, Pickup JC (1993) Serum sialic acid and acute phase protein in type 1 and type 2 diabetes. Clin Chim Acta 219(12): 131-138.

9. Gomes MB, Piccirilo LJ, Nogueira VG, Matos HJ (2003) Acute phase proteins among patients with type1 diabetes. Diabetes Metab 29(4 Pt 1): 405-411.

10. Elia JAD, Weinrauch LA, Gleason RE, Lipinska I, Keough J, et al. (2001) Fibrinogen and factor VII levels improve with glycemic control in patients with type 1 diabetes mellitus who have microvascular complication. Arch Int Med 161(1): 98-101.

11. Defeo P, Volpi E, Lucidi P, Cruciani G, Reboldi G, et al. (1993) Physiological increments in plasma insulin concentration have selective and different effects on synthesis of hepatic proteins in normal humans. Diabetes 42(7): 995-1002.

12. McMillan DE (1989) Increased levels of acute phase serum proteins in diabetes. Metabolism 38(11): 1042-1046.

13. Festa A, D Agostino Jr R, Tracy RP, Haffner SM (2002) Elevated levels of acute phase proteins and plasminogen activator inhibitor 1 predict the development of type 2 diabetes: the insulin resistance atherosclerosis study. Diabetes New York 51(4): 1131-1137.

14. ErnstE, Resch KL (1993) Fibrinogen as a cardiovascular risk factor: A met analysis and review of the of the literature. Annals of Internal Medicine 118(12): 956-963.

15. Barrazzani R, Kiwanuka E, Zanneti M, Cristini M, Vettore M, et al. (2003) Insulin acutely increases fibrinogen production in individuals with type 2 diabetes but not in individuals without diabetes. Diabetes 52(7): 1851-1856.

16. Williams KV, Erbey JR, Becker D, Arslanian S, Ochad TJ (2000) Can clinical factors estimate insulin resistance in Type 1 diabetes. Diabetes 49(4): 626-632.

17. Ehrenwald E, Chisoim GM, Fox PL (1994) Intact human ceruloplasmin oxidatively modifies low density lipoprotein. J Clin Invest 93(4): 14931501.

18. Starkebaum G, Harlan JM (1986) Endothelial cell injury due to copper catalyzed hydrogen peroxide generation from Homocysteine. J Clin Invest 77(4): 1370-1376.

19. Sriharan M, Angela JR, Maria LPO, Bruce BD, Sotes SM, et al. (2002) Total sialic acid and associated elements of the metabolic syndrome in women with and without previous gestational diabetes. Diabetes Care 25(8): 1331-1335.
20. Engstrom G, Stavenow L, Hedblad B, Lind P, Eriksson KF, et al. (2003) Inflammation sensitive plasma protein, diabetes mortality and incidence of myocardial infarction and stroke: A population-based study. Diabetes New York 52(2): 442-447.

21. Schmidt MI, Duncan BB, Sharrett AR, Lindberg G, Savage PJ, et al. (1999) Markers of inflammation and prediction of diabetes mellitus in adult (Atherosclerosis Risk in Communities Study): A Cohort Study. Lancet 353(9165): 1649-1652.

22. Duncan BB, Schmidt MI, Offen BS, Wu KK, Savage PJ, et al. (1999) Factor VIII and other hemostasis variables are related to incident diabetes in adult: The Atherosclerosis Risk in Community (ARIC) study. Diabetes Care 22(5): 767-772.

23. Pradhan AD, Manson JE, Ruai N, Buring JE, Ridker PM (2001) C-reactive protein, interleukin- 6 and risk of developing type 2 diabetes mellitus. JAMA 286(3): 327-334.

24. Hotamisligil GS, Amer P, Cam JF, Atkinson RL, Spiegelman BM (1998) Increased adipose tissue expression of tumor necrosis factor $\alpha$ in human obesity and insulin resistance. J Clin Invest 95(5): 2409-2415.

25. Pickup JC, Crooke MA (1998) Is type 2 diabetes mellitus a disease of the innate immune system? Diabetologia 41(10): 1241-1248.

26. Grimble RF (2002) Inflammatory status and insulin resistance. Curr Opin Cli Nutr Metab 5(5): 551-559.

27. Pradhan AD, Ridkar PM (2002) Do atherosclerosis and type 2 diabetes share a common inflammatory basis? Eur Heart J 23(11): 831-834.

28. Mohammed Ali V, Goodrick S, Rawesh A, Katz DR, Miles JM, et al. (1997) Subcutaneous adipose tissue releases interleukin-6, but not tumor necrosis factor $\alpha$, in vivo. J Clin Endocrinol Metab 82(12): 4196-4200.

29. Hotamisligil GS, Amer P, Cam JF, Atkinson RL, Spiegelman BM (1995) Increased adipose tissue expression of tumor necrosis factor $\alpha$ in human obesity and insulin resistance. J Clin Invest 95(5): 2409-2415.

30. Fried SK, Budkin DA, Greenberg AS (1998) Omental and subcutaneous adipose tissues of obese subject's release interleukin-6: adipose tissue difference and regulation by glucocorticoids. J Clin Endocrinol Metab 83(3): 847-850.

31. Duncan BB, Schmidt MI, Pankow JS, Ballantyne CM, et al. (2003) Low grade inflammation and development of type2 diabetes: The Atherosclerosis Risk in Communities study. Diabetes 52(7): 1799-1805.

32. Bayens JW, Thorpe SR (1999) Role of oxidative stress in diabetic complications: a new perspective on an old paradigm. Diabetes 48(1): $1-9$.

33. Brownlee M (2001) Biochemistry and molecular cell biology of diabetic complication. Nature 414(6865): 813-820.

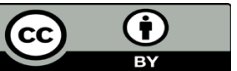

This work is licensed under Creative Commons Attribution 4.0 License

To Submit Your Article Click Here:

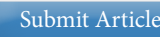

DOI: 10.32474/AD0.2018.01.000121

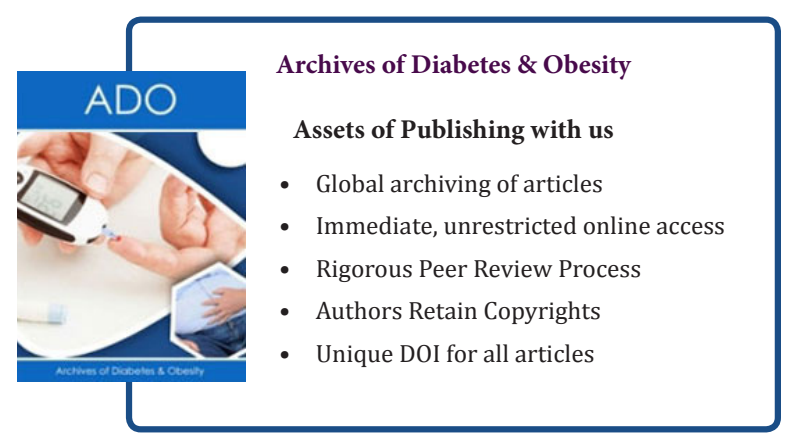

